\title{
NSAID-induced small bowel diaphragms and strictures diagnosed with intraoperative enteroscopy
}

\author{
Douglas A Shumaker MD ${ }^{1}$, Kari Bladen $\mathrm{MD}^{2}$, Ronald M Katon $\mathrm{MD}^{1}$
}

DA Shumaker, K Bladen, RM Katon. NSAID-induced small bowel diaphragms and strictures diagnosed with intraoperative enteroscopy. Can J Gastroenterol 2001;15(9):619-623. Nonsteroidal anti-inflammatory drugs are known to cause mucosal damage in the stomach and duodenum, which may lead to hemorrhage and perforation. However, these medications may also cause damage in the more distal small bowel. Due to the location of these lesions, currently available diagnostic testing may yield false negative results. Two cases of nonsteroidal anti-inflammatory drug-induced small bowel diaphragms presenting as obscure gastrointestinal hemorrhage and recurrent small bowel obstruction, respectively, are discussed. Intraoperative enteroscopy was used to confirm this diagnosis after other diagnostic tests failed to identify the etiology. This procedure may increase the accuracy of exploratory laparotomy in these challenging cases.

Key Words: Intraoperative enteroscopy; Nonsteroidal anti-inflammatory drugs; Obscure gastrointestinal hemorrhage; Small bowel obstruction; Small bowel strictures

\section{Diaphragmes et rétrécissement de l'intestin grêle causés par les AINS et diagnostiqués à l'entéroscopie peropératoire}

RÉSUMÉ : Les anti-inflammatoires non stéroïdiens (AINS), on le sait, causent des lésions à la muqueuse de l'estomac et du duodénum, qui peuvent éventuellement aboutir à des hémorragies et à des perforations. Toutefois, ces médicaments peuvent aussi porter atteinte à l'intégrité de l'intestin grêle distal. Compte tenu du siège des lésions, les examens diagnostiques actuels peuvent donner de faux négatifs. Voici deux cas de diaphragme de l'intestin grêle causé par des AINS se présentant respectivement sous forme d'hémorragie gastro-intestinale obscure et d'occlusion intestinale. L'entéroscopie peropératoire a servi à poser le diagnostic après que les autres examens n'eurent pas permis de cerner l'étiologie. L'intervention peut accroître la fiabilité de la laparotomie exploratoire dans les cas difficiles. astroduodenal mucosal damage is a known side effect J of nonsteroidal anti-inflammatory drugs (NSAIDs) (1). NSAIDs have also been reported to cause lesions in the more distal small bowel and colon. Reported lesions in the small intestine associated with NSAID use have included ulcers, mucosal diaphragms, broad-based strictures and

${ }^{1}$ Division of Gastroenterology, and ${ }^{2}$ Department of Pathology, Oregon Health Sciences University, Portland, Oregon, USA

Correspondence: Dr Ronald M Katon, Division of Gastroenterology, Oregon Health Sciences University, 3181 SW Sam Jackson Park Road,

PV310, Portland, Oregon 97201, USA. Telephone 503-494-8577, fax 503-494-7556, e-mail katonr@ohsu.edu

Received for publication May 18, 2000. Accepted January 31, 2001 


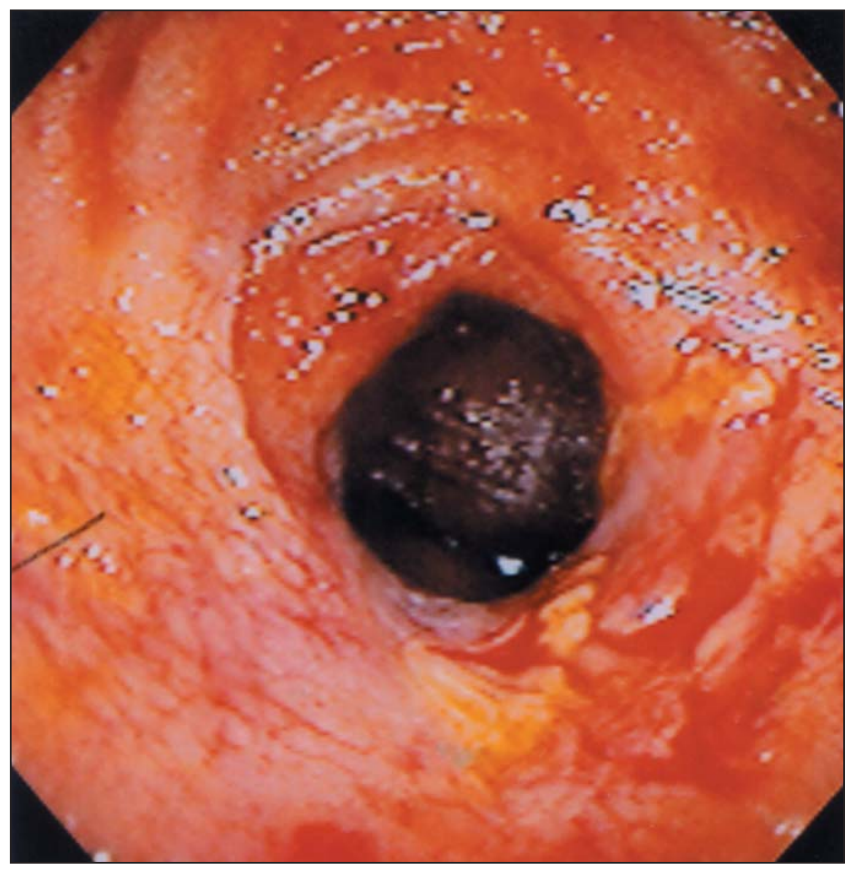

Figure 1) Endoscopic view of nonsteroidal anti-inflammatory druginduced stricture in distal ileum with associated ulceration and active oozing

NSAID enteropathy (2-4). Nonspecific small intestinal ulceration may occur in up to $8 \%$ of patients on long term NSAIDs (5). Although most patients on NSAIDs have no complications, a small percentage may develop iron deficiency anemia, gastrointestinal hemorrhage, or small bowel obstruction or perforation. We report the use of intraoperative enteroscopy to diagnose two cases of small intestinal diaphragm disease and ulceration associated with NSAID use, and review the literature.

\section{CASE PRESENTATION}

Case 1: A 52-year-old man was referred to the authors' institution with recurrent obscure gastrointestinal bleeding and iron-deficiency anemia. He initially presented two years previously with melena and hemoglobin of $0.868 \mathrm{mmol} / \mathrm{L}$. Results of an upper gastrointestinal series with small bowel follow-through and barium enema were unremarkable except for colonic diverticulosis. He had no further melena and responded to iron supplementation with an increase in hemoglobin to $2.015 \mathrm{mmol} / \mathrm{L}$. However, two years later, he experienced recurrent melena associated with a decline in hemoglobin to $1.5 \mathrm{mmol} / \mathrm{L}$. He reported having used ibuprofen ( $600 \mathrm{mg}$ daily) for at least 15 years to control pain associated with osteoarthritis. This medication was discontinued after his first episode of melena, and he denied any current NSAID use. His current medications included iron sulphate and a sedative at bedtime. Esophagogastroduodenoscopy (EGD) revealed no source of the gastrointestinal hemorrhage. Colonoscopy revealed only a few diverticula. He was then referred to the authors' institution, where he underwent a push enteroscopy that revealed a nonobstructing Schatzki's ring, a small hiatal hernia and a normal small bowel to 100 to $120 \mathrm{~cm}$ into the jejunum. A contrast-enhanced computed tomography scan of the abdomen and pelvis revealed multiple hepatic cysts smaller than $1 \mathrm{~cm}$ in diameter, a left renal cyst and sigmoid diverticula. An intraoperative enteroscopy was then performed to the terminal ileum. Three distinct diaphragm-like strictures were identified in a $34 \mathrm{~cm}$ long area of the distal ileum. The most distal lesion had an associated $1 \mathrm{~cm}$ ulcer that was actively oozing at the time of endoscopy (Figure 1). This entire area was resected, along with a single reactive mesenteric lymph node. Pathological evaluation revealed four distinct well-circumscribed ulcerations. The three smaller lesions involved less than $20 \%$ of the internal lumen diameter, with the largest ulcer involving $50 \%$ of the internal diameter. Microscopic evaluation showed four discrete mucosal ulcerations and associated crypt inflammation, thickening of the muscularis mucosae and focal neuronal hyperplasia. Margins of resection were free of inflammation, and the intervening mucosa between ulcerations was normal. No fissures, fistulae or granulomas were identified. These findings were consistent with NSAIDinduced small bowel strictures and ulceration. No further bleeding had occurred at a six-month follow-up.

Case 2: A 49-year-old man was referred to the authors' institution for evaluation of recurrent small bowel obstruction. He reported a total of 26 episodes of partial small bowel obstruction that had responded to conservative treatment over the previous six to seven years. He underwent exploratory laparotomy two years previously with no source of obstruction found, but the jejunum was noted to have an area of active inflammation, and a local reactive mesenteric lymph node was excised. He subsequently underwent an EGD, which revealed a fairly tight postbulbar duodenal stricture that was twice dilated with a balloon dilator to 54 French with good endoscopic results. However, the patient continued to have recurrent episodes of partial small bowel obstruction. Before referral, he underwent a repeat upper endoscopy with a pediatric colonoscope that revealed a widely patent duodenum with no evidence of stricture recurrence and normal findings into the proximal jejunum. In addition, a small bowel follow-through showed no abnormalities. His medical history was significant for an appendectomy as a teenager, rotator cuff injury with arthroscopic surgery two years prior and a motorcycle accident five years prior. He had been using NSAIDs regularly for at least 25 years for various orthopedic injuries. He had been off all NSAIDs for about eight months. He was then referred to the authors' institution, where he initially underwent a push enteroscopy that was normal to approximately $120 \mathrm{~cm}$ beyond the ligament of Treitz. He then underwent an intraoperative enteroscopy that revealed a discrete diaphragm-like stricture approximately $5 \mathrm{~mm}$ in diameter in the distal jejunum with an associated ulceration at one border (Figure 2); the enteroscope could not be passed through this stricture. Two other discrete areas of fibrotic-appearing small bowel were noted on external 


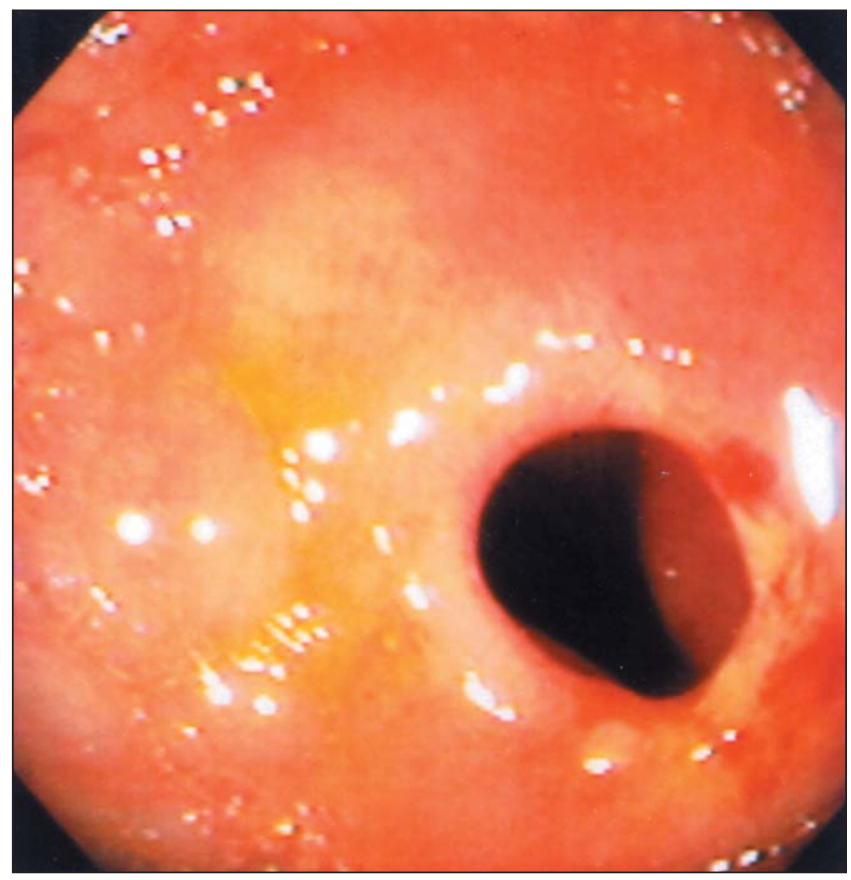

Figure 2) Endoscopic view of nonsteroidal anti-inflammatory druginduced small bowel diaphragm in distal jejunum

examination. A $30 \mathrm{~cm}$ segment of small bowel containing all three areas was resected. The remainder of the small bowel appeared normal. Pathological examination revealed that the area of stricture in the proximal jejunum seen endoscopically contained three successive plicae circulares fused into a thickened circumferential ring covered with granular-appearing mucosa with focal erosion, consistent with an NSAID-induced mucosal diaphragm (Figure 3). In addition, four small (less than $0.8 \mathrm{~cm}$ in diameter), nonobstructing carcinoid tumours were noted in the resected specimen extending through the muscularis propria; all seven lymph nodes were negative for tumour. Two of these tumours were associated with the more distal fibrotic areas palpated by the surgeons. One tumour was near but not involving the mucosal diaphragm. There have been no further bouts of intestinal obstruction at a four-month follow-up.

\section{DISCUSSION}

NSAIDs are among the most widely used drugs due to their potent analgesic, antipyretic and anti-inflammatory properties. The majority of adverse effects of NSAID use occur within the gastrointestinal tract. Gastroduodenal ulceration, hemorrhage, perforation and gastric outlet obstruction are well-documented side effects of NSAID use. It has been estimated that gastroduodenal ulceration occurs in $15 \%$ to $35 \%$ of chronic NSAID users $(5,6)$. However, NSAIDinduced damage can occur throughout the gastrointestinal tract $(2-4)$. The present review focuses on the effect of NSAIDs in the small intestine and the difficulty in diagnosing this effect with standard diagnostic techniques. The utility of intraoperative enteroscopy in making this diagnosis are discussed.
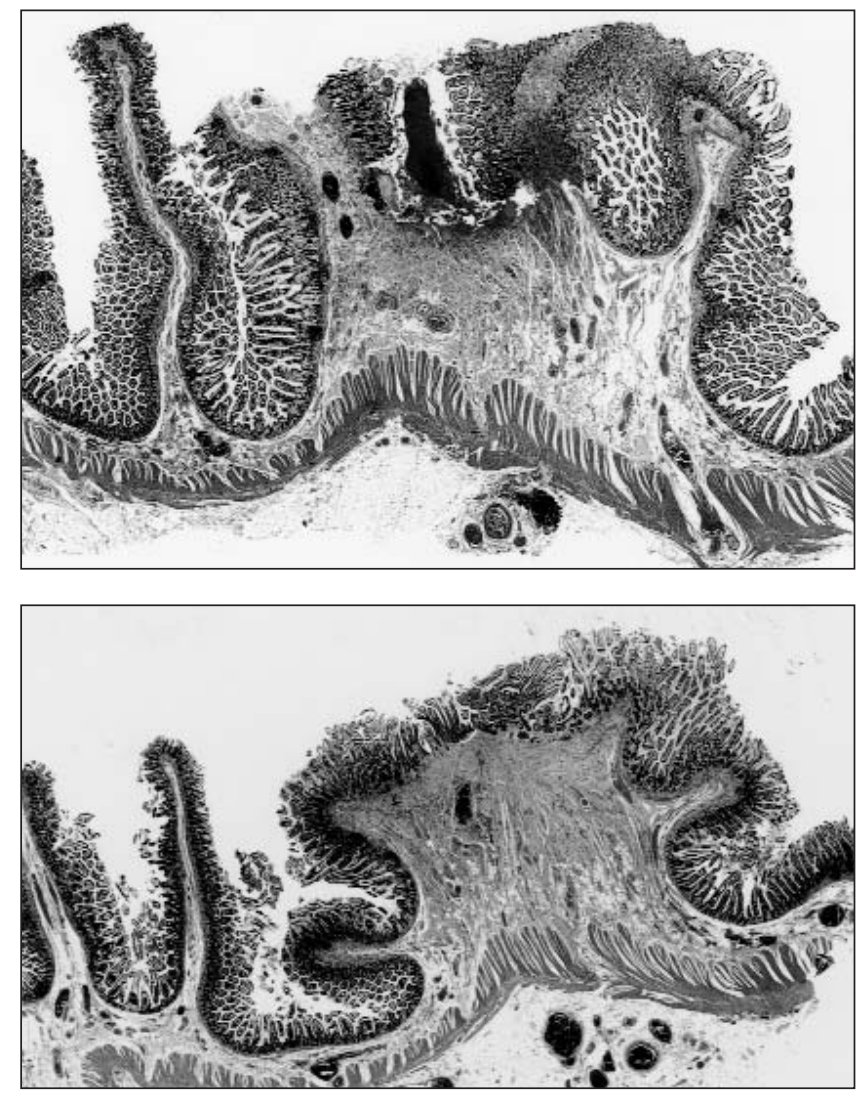

Figure 3) Top Low power photomicrograph of a mucosal diaphragm formed by fusion of several plicae circulares (hemotoxylin and eosin stain, original magnification $\times 10$ ). Bottom Additional section showing focal erosion of mucosa (hemotoxylin and eosin stain, original magnification $\times 12.5$ )

The prevalence and variety of NSAID-induced lesions in the small intestine (jejunum and ileum) have been under-appreciated in the past due to the relative inaccessibility of the small bowel to sensitive diagnostic testing. The reported adverse effects of NSAIDs on the small intestine have included ulcers, strictures and enteropathy. The clinical consequences of these lesions include hemorrhage, perforation, small bowel obstruction, iron-deficiency anemia, protein-losing enteropathy and malabsorption.

Ulceration in the small intestine can lead to hemorrhage or perforation. A common scenario involves a patient with acute gastrointestinal hemorrhage while taking NSAIDs who has no obvious source of bleeding in the stomach, duodenum or colon after routine endoscopic evaluation. As in the first case, the patient is often suspected of having a small bowel source of bleeding. Morris and colleagues (7) in 1991 reported a prospective enteroscopic study of 15 patients with rheumatoid arthritis taking NSAIDs who presented with chronic occult gastrointestinal bleeding. Seven patients $(47 \%)$ were found to have jejunal or ileal ulceration that might explain their chronic blood loss. The authors suggested that small bowel enteroscopy may be a valuable technique for the investigation of obscure gastrointestinal bleeding. Further evidence that NSAIDs cause 
small bowel ulceration comes from an autopsy study of 713 patients (5). Of these patients, 249 had used NSAIDs in the preceding six months. Nonspecific small bowel ulceration was found in $8.4 \%$ of patients who had used NSAIDs compared with only $0.6 \%$ of nonusers. Three patients who were long term NSAID users died of perforated small intestinal ulcers ( $1 \%$ of all deaths in NSAID users). There was no relation between the presence of gastroduodenal ulceration and small bowel lesions, suggesting that the presence or absence of small bowel lesions cannot be predicted based on endoscopic assessment of the stomach and duodenum alone. In a retrospective study of surgical complications of NSAID use in the small intestine, 283 patients who underwent small bowel resection were evaluated (8). Eleven patients (4\%) had 12 small bowel complications associated with NSAID use (defined as daily use of one or more NSAIDs). These complications included bleeding in six patients, perforation in four patients and obstruction in two patients. The ulcer location was variable but occurred most commonly in the terminal ileum (67\%) and at multiple sites in $50 \%$ of patients.

NSAIDs have also been reported to cause strictures in the small intestine, similar to those seen in the esophagus and colon (2-4). These strictures can range from nonspecific, broad-based strictures to intestinal diaphragms (9). Small intestinal diaphragms are considered pathognomonic of NSAID use. They are single or multiple, thin (2 to $4 \mathrm{~mm}$ ), concentric, web-like septa that can narrow the lumen to the size of a pinhole. The true incidence of NSAID-induced small bowel strictures is probably low but uncertain. Lang and colleagues (9) retrospectively reviewed 576 surgically resected small bowel specimens at a single institution. They found seven cases $(1.5 \%)$ of distinct small bowel diaphragms associated with NSAID use (10). The most common clinical consequence of these lesions is small bowel obstruction, as seen in the second case. These lesions are notoriously difficult to diagnose because they may mimic exaggerated plicae circulares during barium studies of the small intestine and usually lie beyond the reach of the standard endoscope. Laparotomy is usually required for their diagnosis, but due to their bland external appearance they may not be apparent on visual inspection (11). Careful palpation of or insufflation of air into the small intestine may increase the likelihood of finding these lesions. There have been two reported cases of small intestinal diaphragms diagnosed by ileoscopy during a colonoscopy $(12,13)$. To our knowledge, the present two cases are the first reported cases of the use of intraoperative enteroscopy in the diagnosis of NSAID-induced small bowel diaphragms and strictures.

The two cases presented here emphasize the difficulty in diagnosing NSAID-induced small bowel diaphragms and ulcers. A high degree of clinical suspicion should be maintained in the patient taking NSAIDs who presents with obscure gastrointestinal bleeding or unexplained small bowel obstruction. Routine upper and lower endoscopy should be performed in all patients with obscure gastroin- testinal bleeding. If these test results are negative, then a small bowel contrast radiographic study (small bowel follow-through or enteroclysis) should be performed. However, the diagnostic yield of a small bowel series is relatively low. It has been estimated that only approximately $5 \%$ of small bowel follow-through examinations detect an intestinal bleeding site (14). Enteroclysis has an increased sensitivity over the standard small bowel follow-through examination, with reported yields of $10 \%$ to $25 \%$ for obscure gastrointestinal bleeding (14). However, enteroclysis is more time consuming, has more side effects and involves more radiation exposure than routine small bowel series. Therefore, many experts still recommend small bowel follow-through examinations as the initial test of choice for suspected small bowel lesions, with enteroclysis reserved for difficult diagnostic dilemmas.

Small bowel enteroscopy has been shown to have a greater yield than barium studies in patients with obscure gastrointestinal bleeding. Reported yields for push enteroscopy when evaluating patients with obscure gastrointestinal bleeding have varied from $13 \%$ to $64 \%$ (14). However, current technology limits the depth of insertion of push enteroscopy. Therefore, enteroclysis is often added to the evaluation of the patient with suspected small bowel lesions. If all of the aforementioned studies are negative and the patient continues to experience symptoms from suspected NSAID-related small bowel lesions, operative intervention is often necessary. However, as demonstrated in the present cases, small bowel diaphragms and ulcerations can be quite subtle and frequently overlooked during a routine external evaluation of the small bowel at operation. Intraoperative enteroscopy, considered to be the ultimate diagnostic procedure for complete evaluation of the small bowel, should be considered whenever the other methods of investigation fail to uncover an etiology for a suspected small bowel lesion that results in transfusion-dependent gastrointestinal bleeding or recurrent obstruction (10). In the future, virtual enteroscopy (computed tomography or magnetic resonance imaging) (10) or wireless endoscopy (15) may allow less invasive and more accurate evaluation of the small intestine.

\section{SUMMARY}

NSAID-induced damage in the small intestine may manifest as ulceration, strictures and enteropathy. These lesions should be considered in any patient who has taken NSAIDs and presents with acute obscure gastrointestinal hemorrhage, small bowel obstruction, or chronic occult gastrointestinal blood loss and iron-deficiency anemia. Results of routine endoscopic evaluation of the stomach, duodenum and colon are often negative in this setting and do not predict the presence or absence of small bowel lesions. In addition, radiographic assessment of the small intestine is usually not diagnostic. A high index of suspicion and the judicious use of endoscopic techniques, including push enteroscopy and intraoperative enteroscopy, may permit an accurate diagnosis in many cases. 


\section{REFERENCES}

1. Soll AH. Non-steroidal anti-inflammatory drugs and peptic ulcer disease. Ann Intern Med 1991;114:307-19.

2. Cryer B, Kimmey MB. Gastrointestinal side effects of non-steroidal anti-inflammatory drugs. Am J Med 1998;105:20S-30S.

3. Bjarnason I, Hayllar J, MacPherson AJ, et al. Side effects of non-steroidal anti-inflammatory drugs on the large and small intestine. Gastroenterology 1993;104:1832-47.

4. Bjarnason I, MacPherson AJS. Intestinal toxicity of non-steroidal anti-inflammatory drugs. Pharmacol Ther 1994;62:145-57.

5. Allison MC, Howatson AG, Torrance CJ, et al. Gastrointestinal damage associated with the use of nonsteroidal antiinflammatory drugs. N Engl J Med 1992;327:749-54.

6. Zuckerman MJ, Hernandez JA, Marwah RK, et al. Prevalence of upper gastrointestinal lesions in patients taking chronic nonsteroidal anti-inflammatory drug therapy. Am J Gastroenterol 1997;92:363-4

7. Morris AJ, Madhok R, Sturrock RD, et al. Enteroscopic diagnosis of small bowel ulceration in patients receiving non-steroidal antiinflammatory drugs. Lancet 1991;337:520.

8. Kessler WF, Shires T, Fahey TJ. Surgical complications of nonsteroidal antiinflammatory drug-induced small bowel ulceration. J Am Coll Surg 1997;185:250-4.

9. Lang J, Price AB, Levi AJ, et al. Diaphragm disease: pathology of disease of the small intestine induced by non-steroidal antiinflammatory drugs. J Clin Pathol 1988;41:516-26.

10. Delmotte JS, Gay G, Houcke P, Mensard Y. Intraoperative endoscopy. Gastrointest Endosc Clin North Am 1999;9:61-9.

11. Bjarnason I, Price AB, Zanelli G, et al. Clinicopathologic features of nonsteroidal antiinflammatory drug-induced small intestinal strictures. Gastroenterology 1988;94:1070-4.

12. Hershfield NB. Endoscopic demonstration of non-steroidal anti-inflammatory drug-induced small intestinal strictures. Gastrointest Endosc 1992;38:388-90.

13. Cutler C, Rex DK, Cummings OW. Per anus enteroscopic demonstration of a non-steroidal anti-inflammatory drug-induced ileal stricture. Gastrointest Endosc 1993;39:601-3.

14. Lewis BS. Radiology versus endoscopy of the small bowel. Gastrointest Endosc Clin North Am 1999;9:13-27.

15. Swain P. Wireless endoscopy. Gastrointest Endosc 2000;51:AB143. (Abst 4467) 


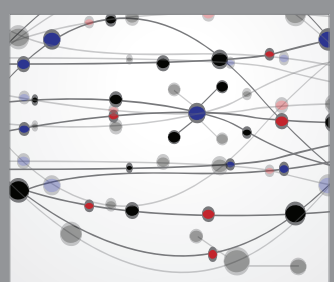

The Scientific World Journal
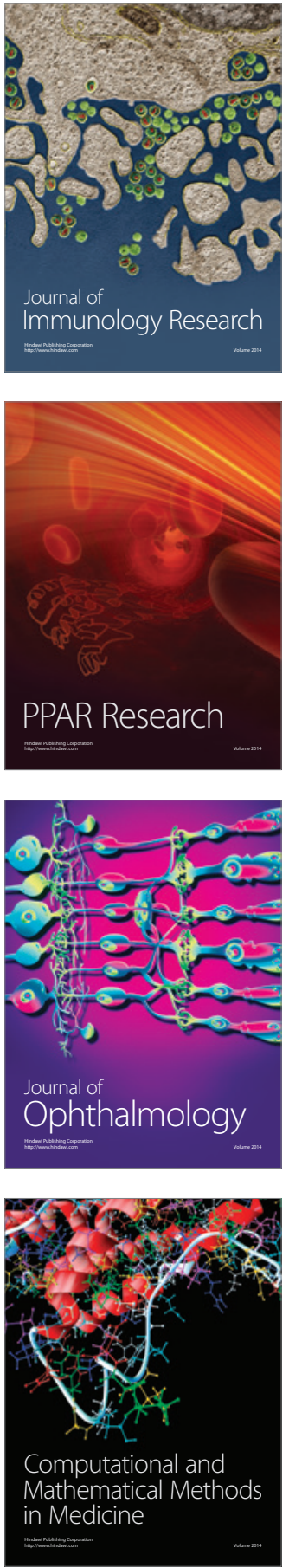

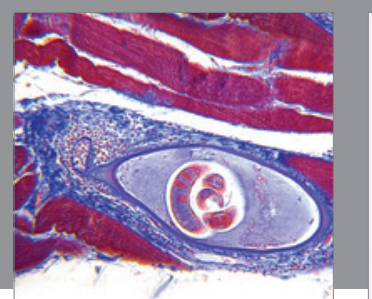

Gastroenterology Research and Practice

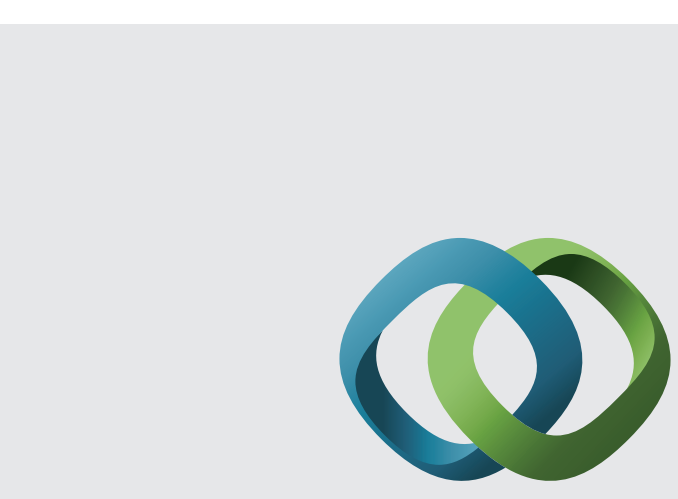

\section{Hindawi}

Submit your manuscripts at

http://www.hindawi.com
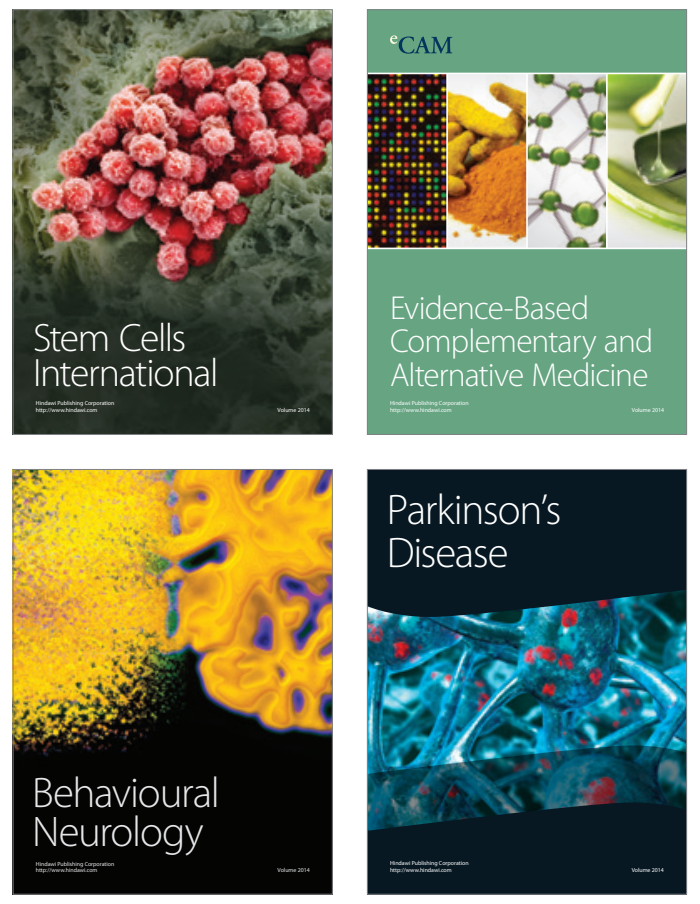
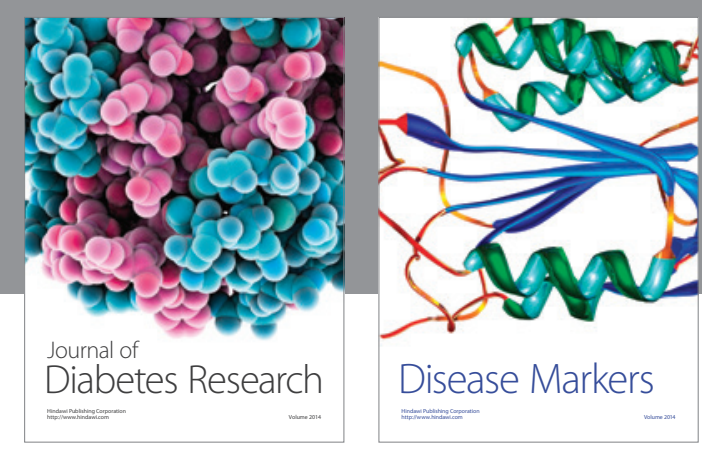

Disease Markers
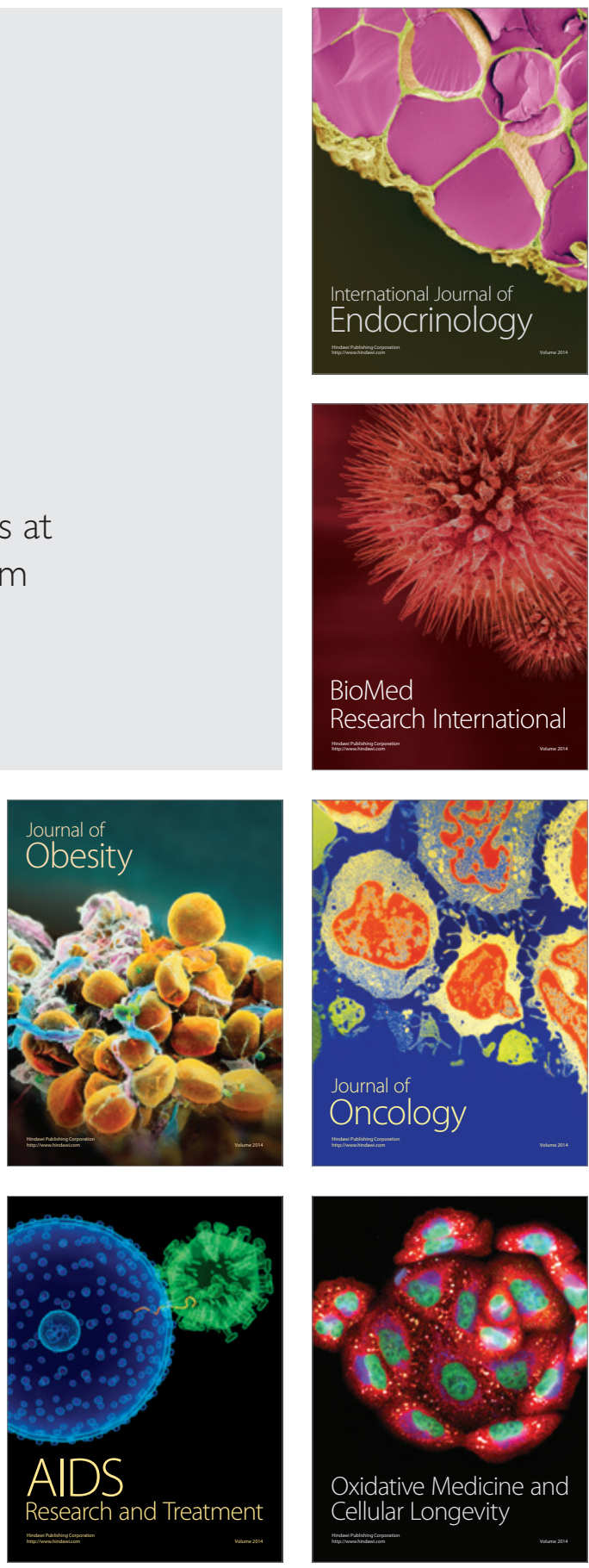